\title{
Guests Editors' Editorial Note on Special Issue of Advances in Cellular Automata Modeling
}

Starting from the previous century up to today, various modeling prototypes with unique features, special characteristics, and well-established computation abilities have been introduced to the scientific society as tentative computation alternatives to differential and partial differential equations used to describe physical systems and processes. Among them, a model originally conceived by famous John von Neumann, namely Cellular Automata (CA), due to its inherent impressive abilities managed to provide scientists and practitioners with an efficient modeling framework for a variety of applications, very often of interdisciplinary nature. Indeed, although the CA field is considered to be a relatively old and established one, these simple but powerful systems and their most recent variations that simulate macroscopic processes from numerous elementary and local interactions continue to attract the interest of researchers after their original introduction. Using the words of Nobelist Richard Feynman, "CA are very effective in simulating physical systems and solving scientific problems, because they can capture the essential features of systems where global behaviour arises from the collective effect of simple components which interact locally."

In this context, in the last decades, the International Conference on Cellular Automata for Research and Industry (ACRI) series of conferences has been an internationally renowned forum for all those interested in the theory and applications of CA, including CA tools and computational sciences. It is also concerned with applications and solutions of problems from the fields of arts, biology, chemistry, communication, cultural heritage, ecology, economy, geology, engineering, computer science, medicine, physics, sociology, and so on, allowing scholars coming from different studies and experience in CA to discuss and merge their competencies and results, to identify new issues, and to enlarge the research fields of CA. Taking the initiation from the 10th edition of the ACRI 2012 Conference that took place in Santorini Island, Greece, in September 2012, an open call was announced for the submission of papers either related with the CA papers of the ACRI 2012 Conference or unpublished high-quality works strongly related to the modeling aspects of CA in any field of physics, engineering, environment science, social science, and life science. As a result, we finally ended with four intriguing and mostly CA-based papers that constitute this Special Issue of Advances in Cellular Automata Modeling in ACM TOMACS.

The first article, entitled "Morphological Coevolution for Fluid Dynamical-Related Risk Mitigation” by Filippone et al., presented a comprehensive study of CA modeling based on an evolutionary approach for devising protective measures to divert lava flows.

Authors' addresses: S. Bandini and G. Vizzari, Department of Computer Science, Systems \& Communication, University of Milano-Bicocca, Viale Sarca 336 - U14 20126 Milano, Italy; emails: \{bandini, viz\}@disco. unimib.it; G. Ch. Sirakoulis, Department of Electrical and Computer Engineering, Democritus University of Thrace, Xanthi 67100, Greece; email: gsirak@ee.duth.gr.

Permission to make digital or hard copies of part or all of this work for personal or classroom use is granted without fee provided that copies are not made or distributed for profit or commercial advantage and that copies show this notice on the first page or initial screen of a display along with the full citation. Copyrights for components of this work owned by others than ACM must be honored. Abstracting with credit is permitted. To copy otherwise, to republish, to post on servers, to redistribute to lists, or to use any component of this work in other works requires prior specific permission and/or a fee. Permissions may be requested from Publications Dept., ACM, Inc., 2 Penn Plaza, Suite 701, New York, NY 10121-0701 USA, fax +1 (212) 869-0481, or permissions@acm.org.

(c) 2016 ACM 1049-3301/2016/01-ART17 $\$ 15.00$

DOI: http://dx.doi.org/10.1145/2856511 
In specific, an evolutionary computation-based decision support system for defining and optimizing volcanic hazard mitigation interventions was introduced. Furthermore, the SCIARA-fv2 Cellular Automata numerical model has been applied for simulating lava flows at Mt. Etna (Italy) volcano while a Parallel Genetic Algorithm (PGA) adopted for optimizing protective measures construction by morphological evolution and run in General-Purpose Graphic Processing Units (GPGPUs). A Single Barrier (SBA) approach was then originally planned and combined with a GA component, called Evolutionary Greedy Strategy (EGS), implemented by introducing multibarrier protection measures in order to improve the efficiency of the final solution; finally, a Coevolutionary Cooperative Strategy (CCS) was introduced where all barriers are encoded in the genotype and because all the constituents parts of the solution interact with the GA environment, a mechanism of cooperation between individuals has been favored. These exact solutions provided by CCS were eventually extremely efficient and, in particular, the devised protection work total volume used for flow diversion, avoiding that the lava reaches the inhabited area. Consequently, the study resulted in quite positive results and was successfully introduced as the first application of morphological evolution for lava flow mitigation.

The work by Tsompanas et al. entitled "Modeling Cache Memory Utilization on Multicore Using Common Pool Resource Game on Cellular Automata" introduced a parallel model for modeling the utilization of on-chip memory, based on CA and game theory. The utilization of memory is an important factor, as it is proven to correlate with the efficiency of a computing system. Namely, every core in the multicore system is simulated by a cell on a CA lattice in order to extract the parallel dynamics of the CA. Another advantage of CA is the definition of the radius of the neighborhood and their ability to incorporate inhomogeneities; that makes the model able to cope with the increasing diversity of cache memory hierarchy on modern processors. Having in mind the inherent parallel nature of CA fully exploited when its rule is implemented on hardware, the result can serve as a co-processor or a hardware accelerator, increasing the efficiency of a modern multicore system. Furthermore, game theory is used to incorporate the local rule of the CA; in specific, the common pool resource $(\mathrm{CPR})$ game is selected, as it sufficiently captures the dynamics of multiple players interacting under the same rules while conflicting for the utilization of a common good. Consequently, a core of the system will be modeled by a CA cell whose local rule is defined by game theory concepts. As a result, when acquiring data from hardware performance counters and processing them with the proposed model online, the performance of the system can be calculated in real time and a better scheduling strategy can be adopted. More specifically, the results produced by the CA-based model are verified by real system profiling experiments. More specifically, the model is able to simulate the speedup achieved by an increased number of cores available to the system, based on the cache memory hit and miss counts with accuracy of $3.2 \%$ to $10.9 \%$.

Reppas et al. discuss the extrinsic noise effect on lateral inhibition differentiation waves in their article entitled "The Extrinsic Noise Effect on Lateral Inhibition Differentiation Waves." In this context, they suggest a general framework of the effect of extrinsic noise in the Notch/Delta induced cellular pattern formation. In particular, they adopted a simplified version of the model described by Collier et al. (1996), which can produce a "salt-and-pepper" cellular pattern through the use of a CA formulation. Furthermore, the impact of the differentiation wave associated with the Notch/Delta mechanism was investigated on the cell fate specification for the stochastic and deterministic case. Results showed that in the deterministic case, pattern formation is coordinated by a wave of differentiation, whereas on the stochastic case, extrinsic noise weakens the effect of the differentiation wave. The provided analysis presented that 
even small noise intensities can give rise to "frustrated cell furrows." However, the observation of "frustrated states" seems to be more an exception than a rule in biological development, while obviously, cells have developed de-noising mechanisms, noise related originally with negative effects as something undesirable. In biological systems, it represents "randomness"; thus, it may well be regarded as something desirable. In conclusion, in this article, a stochastic CA with continuous state space was used to exhibit that extrinsic noise results in the emergence of steady-state furrow patterns of cells in a "frustrated/transient" phenotypic state.

Finally, in their article entitled "PAM: Particle Automata in Modeling of Multi-scale Biological Systems," Dzwinel et al. present a new concept of modeling framework, which, while arriving from CA, integrates particle method (PM) with graph dynamical systems (GDS), called the particle automata model (PAM). The main concept and the corresponding assumptions, like that mechanical response of a macroscopic system on internal or external stimuli can be simulated by the spatio-temporal dynamics of a graph of interacting particles representing fine-grained components of a biological tissue. The latter could be (biological) cells, clusters of cells, or microtissue fragments whose representation and management by the PAM model are presented in details. More specifically, the authors have shown that PAM reflects a specific coarse-graining procedure in which the microscopic degrees of freedom are encapsulated inside a particle and manifest in changes of its state vector, consequently, influencing local and global dynamics of the entire system. This way, the PA ensemble can simulate multiscale systems by using only one macroscopic spatiotemporal scale controlled by particle dynamics and microscopic rule-based operator. Finally, in order to help the potential reader to better appreciate the proposed approach, three models of very different biological phenomena, namely blood clotting, tumor proliferation, and fungal wheat infection, are presented in the context of the PA modeling method. Consequently, the generic and flexible modeling framework, although with reasonable computational complexity provided by PAM, may contribute to more intuitive and faster development of computational models of complex multiscale biological processes.

This special issue would not have been possible without the support and collaboration of many people that helped in different ways to make it true. First, we do wish to thank all the contributing authors for their hard and inspiring work both in writing and adequately presenting their ideas and insights on the possible usage of CA modeling aspects to various and interdisciplinary applications as well for revising their articles according to the EiC's and reviewers' prompt. We also thank the numerous reviewers for their referring services that enabled us through their continuous efforts to make this special issue come true. Finally, we thank ACM TOMACS's Editor-in-Chief Adelinde Uhrmacher for her support throughout the overall process of definition and management of the special issue.

Stefania Bandini

University of Milano-Bicocca Georgios Ch. Sirakoulis

Democritus University of Thrace

Giuseppe Vizzari

University of Milano-Bicocca

Guests Editors 